\title{
A Comparative Study of the Recurrence Rate in Hypofractionated versus Conventional Postmastectomy Radiation in Breast Cancer
}

\author{
Aravindh Sivanandan Anand', Abul Hussain Mirsa1, Amina Basheer ${ }^{2}$ \\ ${ }^{1}$ Department of Radiation Oncology, Government Medical College, Trivandrum, India \\ ${ }^{2}$ Department of Pharmacology, Government Medical College, Trivandrum, India \\ Email: anandrt2006@yahoo.com, abulhussainmklm@gmail.com
}

How to cite this paper: Anand, A.S, Mirsa, A.H. and Basheer, A. (2021) A Comparative Study of the Recurrence Rate in Hypofractionated versus Conventional Postmastectomy Radiation in Breast Cancer. Journal of Cancer Therapy, 12, 736-750. https://doi.org/10.4236/jct.2021.1212064

Received: November 24, 2021

Accepted: December 24, 2021

Published: December 27, 2021

Copyright $\odot 2021$ by author(s) and Scientific Research Publishing Inc. This work is licensed under the Creative Commons Attribution International License (CC BY 4.0).

http://creativecommons.org/licenses/by/4.0/

(c) (i) Open Access

\begin{abstract}
Background: Treatment options available for breast cancer are Surgery [Breast conservation surgery (BCS) or Modified radical mastectomy (MRM)]; Radiation treatment [Conventional radiation treatment or CRT and Hypofractionated radiation treatment or HRT] and Chemotherapy. In the postmastectomy or post lumpectomy setting, radiotherapy (RT) improves loco-regional control. CRT for breast includes $50 \mathrm{~Gy}$ in 25 fractions (2 Gy per fraction) and HRT includes $42.5 \mathrm{~Gy}$ in 16 fractions (2.7 Gy per fraction) or extreme hypofractionation like $26 \mathrm{~Gy}$ in 5 fractions. Alpha/beta value for breast is less, so HRT is ideal for breast. So, there will be good loco-regional control, without increased normal tissue damage. This study aims to identify recurrence rate and toxicity in breast cancer patients treated using conventional and hypofractionated postmastectomy radiotherapy among Indian population. Primary objective: To assess recurrence rate of disease in breast cancer patients treated using hypofractionated postmastectomy radiation and to compare it with breast cancer patients treated using conventional postmastectomy radiation. Secondary objective: To assess the toxicity in hypofractionation and conventional fractionation arm. Methods: This is a prospective observational study conducted in Department of Radiation Oncology from December 2017 to June 2019. Radically treated breast cancer patients who received radiation treatment either conventional or hypofractionated as one of the treatment modalities were included in the study. Data were collected using a structured proforma, history and physical examination, master file of the patients, lab results and the imaging reports, written informed consent form, ECOG performance status scale (Eastern Cooperative Oncology Group), RTOG (Radiation Therapy Oncology Group) Acute Radiation Morbidity Scoring Schema. Patients were monitored for 18 months to identify recurrence rate and toxicity in each arm. Results and discussion: A total of
\end{abstract}


241 patients were enrolled into this study, among them 175 patients $(73 \%)$ were given hypofractionation radiotherapy and 66 patients (27\%) were given conventional radiotherapy. In hypofractionation arm, recurrence was found in 14 patients (8\%), of which, 3 were local recurrences [chest wall] and 11 were systemic recurrences, while in conventional arm, recurrence was found in 4 patients $(6 \%)$ and all of them were systemic recurrences. Recurrence rate in hypofractionation arm was $8 \%$ and in conventional arm was $6.10 \%$. The Kaplan Meier curve shows no significant difference between the two arms with $\mathrm{p}$ value $=0.76$. Acute toxicities assessed were dermatitis, esophagitis and pneumonitis. Among acute dermatitis, 4 patients had grade 3 and 2 patients had grade 4 in hypofractionation arm, while in conventional arm, 7 patients had grade 3 and 1 patient had grade 4 . Grade 1 and 2 together versus grade 3 and 4 acute dermatitis showed a statistically significant difference between the two arms, with more acute toxicity in the conventional arm. Among acute esophagitis, 1 patient had grade 3 and no patients had grade 4 in hypofractionation arm; while in conventional arm, no grade 3 and grade 4 acute esophagitis were found. Among acute pneumonitis, 2 patients had grade 3 and 1 patient had grade 4 in hypofractionation arm, while in conventional arm, 1 patient had grade 3 and no patients with grade 4 were found. Grade 2 and grade 3 acute lung toxicities were found in patients with central lung distance more than $1.5 \mathrm{~cm}$. Conclusion: It was found that the recurrence rate of hypofractionation radiotherapy was comparable to conventional fractionation radiotherapy. With respect to acute dermatitis, grade 1 and grade 2 were significantly more in conventional than hypofractionation arm. Hypofractionated radiotherapy is an equally effective option to conventional radiotherapy and should be encouraged, especially for developing countries like India where the resource is limited, and the incidence of tumour is high.

\section{Keywords}

Breast Cancer, Post Mastectomy Radiation, Conventional, Hypofractionation, Recurrence Rate, Acute Toxicity

\section{Introduction}

Breast cancer is a major public health problem for women throughout the world. It remains the most frequent cancer in women and second most frequent cause of cancer death. The adoption of screening mammography, use of adjuvant and neoadjuvant therapy and hormone therapy have made drastic change in outcome of treatment for the past few decades [1].

It is the most common cancer in women both in developed and less developed countries. Carcinoma breast has ranked number one cancer among Indian females with age adjusted rate as high as 25.8 per 100,000 women and mortality 12.7 per 100,000 women. There is a significant increase in incidence and cancer-associated morbidity and mortality in Indian subcontinent as described in global and Indian studies. The standard of care for carcinoma breast is multi- 
modality, which is surgery [either breast conservation surgery (BCS) or modified radical mastectomy (MRM)], either neoadjuvant or adjuvant chemotherapy depending on the stage at diagnosis, followed by radiotherapy treatment [Conventional RT (CRT) or Hypo fractionated RT (HRT)] as indicated since there is a higher incidence of local recurrence; followed by hormonal therapy depending on receptor status and menstrual status. Many studies have confirmed the rationale for post mastectomy radiation (PMRT), which is the prevention of a loco-regional recurrence. The theoretical idea is that, clinically occult persistent disease may be present in operative site and draining nodes which may act as a source of metastases, thus targeting operative site through radiotherapy which may have a beneficial effect on the patients. In the post mastectomy or post lumpectomy setting, radiotherapy improves loco-regional coGy/fraction ntrol and overall survival [2]-[12]. The conventional fraction regimen $(1.8-2)$ which is followed for adjuvant radiation treatment was to deliver $50 \mathrm{~Gy}$ in 25 fractions over a 5 -week time period, with 2 Gy per fraction each day. Those who had BCS would further require a boost of 10 - 15 Gy delivered with 2 Gy in each fraction, extending the treatment time for 2 more weeks. As the understanding of radiobiology of breast cancers grew, the concept that hypofractionation would be beneficial for breast cancers evolved. The $\alpha / \beta$ ratio of breast cancer is $3-4 \mathrm{~Gy}$, similar to late reacting tissues. Since the $\alpha / \beta$ value for breast is less, hypofractionation is ideal for breast. The hypo fractionated regimen ( $>2.2 \mathrm{~Gy} /$ fraction) is assessed in various studies and has demonstrated equivalent tumour control and cosmetic outcome with shorter courses of therapy, without increased normal tissue damage [2]-[12]. Consequently, this approach was adopted into routine practice in many centres.

\section{Methodology}

This study was conducted in the Department of Radiotherapy and Oncology of Government Medical College, Trivandrum in State of Kerala, India. This institution is a tertiary cancer centre of the State. This study was approved by the scientific Institutional Review Board and by the Human ethical committee. The primary objective is to assess recurrence rate of disease in breast cancer patients treated using hypofractionated postmastectomy radiation and to compare it with breast cancer patients treated using conventional postmastectomy radiation. The secondary objective is to assess the toxicity in hypofractionation and conventional fractionation arm. This is a single institutional prospective observational study (longitudinal) of all female patients who were diagnosed to have carcinoma breast and attended radiotherapy OPD for postmastectomy radiation treatment in the Department of Radiotherapy, Government Medical College, Thiruvananthapuram, during the study period, from December 2017-June 2019. This study period represents 18 months from the date of ethical committee clearance. This study included all consecutive female patients reporting for treatment during this period and willing to participate in the study with in- 
formed consent. Histologically confirmed cases of carcinoma breast treated surgically using MRM, ECOG performance status of 0 to 2, age more than 18 years and less than 75 years, normal bone marrow, renal and liver function, all node positive disease, all margin positive disease, T3 or T4 disease. Patients not willing for the study; history of any other malignancy; history of collagen vascular disease; history of SLE; history of previous chest wall irradiation were excluded. Data collection was done using structured proforma, history and physical examination, master file of the patients, lab results and the imaging reports, written informed consent form, ECOG performance status scale (Eastern Cooperative Oncology Group) and RTOG Acute Radiation Morbidity Scoring Schema. Patients registered in RT department as breast cancer was checked for eligibility criteria and enrolled for the study. Clinical, diagnostic and staging evaluation was done by the consulting physician and recorded. Systemic chemotherapy that the patient received was noted (AC $\times 4$ cycles $\rightarrow$ Docetaxel $\times 4$ cycles or TAC $\times 6$ cycles). Radiation treatment planned by the radiation oncologist (Hypofractionation or Conventional fractionation) was noted. All radiation parameters including the field size, inter field distance (IFD), central lung distance (CLD) was recorded. Radiation dose received by the patient was recorded and weekly toxicity was assessed using RTOG toxicity criteria. Patient was followed up as per the ongoing departmental protocol (during treatment weekly, after that monthly till $1^{\text {st }}$ visit, followed by 3 monthly for up to the next 18 months). Locoregional or systemic metastasis was noted in the prepared proforma for each patient. Data analysis was done using Excel 2010 and SPSS 18 statistical software. Each variable analysed in this study were entered in Excel 2010 work sheet. The continuous variables were described by the median and/or the mean \pm standard deviation. The qualitative variables were described by the distribution of their modalities. The groups in question were compared using Student's test for continuous variables and by Pearson's $\mathrm{Chi}^{2}$ test for qualitative variables. Also, ANOVA test was used in analysis, to find out the relationship between CLD and acute pneumonitis. All statistical tests were two-sided, and $p$ value $\leq 0.05$ was considered to be statistically significant. The highest toxicity during any 4 week of radiation therapy was considered as toxicity grade of that patient.

\section{Observations and Results}

In this prospective observational study, we have enrolled a total of 241 pathologically proved female patients with carcinoma breast who satisfied the selection criteria. Among this, 175 postmastectomy patients were treated with adjuvant hypofractionation schedule and 66 patients were treated with adjuvant conventional fractionation (Tables 1-4 and Figure 1).

\section{Discussion}

The median age in hypofractionation arm was 54 years and in conventional fractionation arm was 48 years. This was similar to the findings of the study conducted 
Table 1. Baseline characteristics of the patients.

\begin{tabular}{lcccc}
\hline \multirow{2}{*}{ Variable } & \multicolumn{2}{c}{ Hypofractionation arm } & \multicolumn{2}{c}{ Conventional arm } \\
\cline { 2 - 5 } & Frequency & Percentage & Frequency & Percenta \\
\hline $\begin{array}{l}\text { 1) Age of the patient } \\
<40\end{array}$ & 16 & & & \\
$40-50$ & 52 & 29.7 & 10 & 15 \\
$50-60$ & 62 & 35 & 27 & 41 \\
$60-70$ & 41 & 23 & 5 & 7.5 \\
$>70$ & 4 & 2.3 & 4 & 6
\end{tabular}

2) ECOG PS

0

1

2

3) Comorbidities

Diabetes mellitus

Hypertension

Coronary artery disease

4) Main presentation

Lump

Axillary swelling

Nipple retraction

Nipple discharge

Pain in the breast

5) Menopause

Premenopausal status

Perimenopausal status

Postmenopausal status

6) Marital status

Married

Unmarried

7) Parity

0

1

2

3

4

5

8) History of OCP use

No OCP use

With OCP use
39

123

13

37

48

7

171

1

1

1

1

31

20

124

174

1

2

13

132

23

3

2

173
22

70

7

21.1

27.4

4

19.7

21.2

$3 \quad 4.5$

97.7

0.6

0.6

0.6

0.6

$1 \quad 1.5$
30.3

19.7

50

$99.4 \quad 64 \quad 97$

$\begin{array}{lll}0.6 & 2 & 3\end{array}$

$\begin{array}{ccc}1.1 & 4 & 6.1 \\ 7.4 & 3 & 4.5 \\ 75.4 & 52 & 78.8 \\ 13.1 & 4 & 6.1 \\ 1.7 & 2 & 4 \\ 1.1 & 1 & 1.5\end{array}$

98.9

66

$100 \%$

5

1

0

5

6




\section{Continued}

\section{9) Breastfeeding history}

Adequate breastfeeding (at least 1 year)

172

98.3

62

93.9

No breastfeeding

3

1.7

6.1

10) Family history of malignancy

(i) No history

(ii) Breast cancer one first degree relative one second degree relative one third degree relative
(iii) Ovarian cancer
(iv) Uterine cancer
(v) Brain cancer
(vi) other malignancy

ative one third

$\begin{array}{cccc}7 & 4 & 5 & 7.6 \\ 5 & 2.9 & 1 & 1.5 \\ 1 & 0.6 & - & - \\ 2 & 1.1 & 1 & 1.5 \\ 3 & 1.7 & - & - \\ 5 & 2.9 & - & - \\ 7 & 4 & 5 & 7.6\end{array}$

Table 2. Tumour characteristics.

\begin{tabular}{|c|c|c|c|c|}
\hline \multirow{2}{*}{ Variable } & \multicolumn{2}{|c|}{ Hypofractionation arm } & \multicolumn{2}{|c|}{ Conventional arm } \\
\hline & Frequency & Percentage & Frequency & Percentage \\
\hline
\end{tabular}

1) Side of the tumour

$\begin{array}{lcccc}\text { Right } & 74 & 42.3 & 32 & 48.5 \\ \text { Left } & 101 & 57.5 & 34 & 51.5\end{array}$

2) Tumour quadrant

$\begin{array}{lcccc}\text { UOQ } & 140 & 80 & 55 & 82 \\ \text { Paracentral } & 13 & 7.6 & 5 & 7.5 \\ \text { UIQ } & 8 & 4.6 & 2 & 3 \\ \text { LOQ } & 8 & 4.6 & 3 & 4.5 \\ \text { LIQ } & 4 & 2.3 & 1 & 1.5\end{array}$

\section{3) Tumour status}

$\begin{array}{ccccc}\text { Tx } & 6 & 3.4 & 3 & 4.5 \\ \text { T1 } & 12 & 6.9 & 1 & 1.5 \\ \text { T2 } & 75 & 42.9 & 28 & 42.4 \\ \text { T3 } & 50 & 28.6 & 23 & 34.8 \\ \text { T4 } & 32 & 18.3 & 11 & 16.7\end{array}$

4) Nodal status

$\begin{array}{lcccc}\text { Nx } & 5 & 2.9 & 3 & 4.5 \\ \text { N0 } & 42 & 24 & 15 & 22.7 \\ \text { N1 } & 69 & 39.4 & 24 & 36.4 \\ \text { N2 } & 40 & 22.9 & 16 & 24.2 \\ \text { N3 } & 19 & 10.9 & 8 & 12.1\end{array}$




\section{Continued}

5) Composite stage

$\begin{array}{lcccc}\text { I } & 2 & 1.1 & - & - \\ \text { II } & 70 & 40 & 21 & 31.8 \\ \text { III } & 95 & 54.3 & 39 & 59.1 \\ \text { Could not be assessed } & 8 & 4.6 & 6 & 9.1\end{array}$

6) Mammogram

$\begin{array}{lcccc}\text { BIRADS 2 } & 3 & 1.7 & - & - \\ \text { BIRADS 3 } & 5 & 2.9 & - & - \\ \text { BIRADS 4 } & 28 & 16 & 16 & 24.2 \\ \text { BIRADS 5 } & 135 & 77.1 & 49 & 74.2 \\ \text { BIRADS 6 } & 4 & 2.3 & 1 & 1.5\end{array}$

7) Histology

IDC

Metaplastic carcinoma

166

95

Papillary carcinoma

Lobular invasive carcinoma

2.3

Secretory carcinoma

0.6

8) Tumour grade

1

2

3

9) Other characteristics

Lymphovascular space invasion

Perineural invasion

Base of resection positive

Margins positive

10) Receptor status

ER positive
PR positive
HER2-neu positive

5

145

25

19

4

32

17

7

80

51

43
2.9

83

14.3
95.5

4.2

.2

1.5

$9 \quad 13.6$

Table 3. Treatment characteristics.

\begin{tabular}{lcccc}
\hline & Variable & Hypofractionation arm & \multicolumn{2}{c}{ Conventional arm } \\
\cline { 3 - 5 } & Frequency & Percentage & Frequency Percentage \\
\hline 1) Chemotherapy & & & & \\
Neoadjuvant & 46 & 26.3 & 16 & 24.2 \\
Adjuvant & 129 & 73.7 & 50 & 75.8 \\
\hline
\end{tabular}




\section{Continued}

2) Chemotherapy schedule

$$
\begin{aligned}
& \text { AC } \times 4 \rightarrow \text { Docetaxel } \times 4 \\
& \text { AC } \times 4 \rightarrow \text { Paclitaxel } \times 4 \\
& \text { TAC } \times 6
\end{aligned}
$$

No chemotherapy

\section{3) Radiation planning details}

(i) Supraclavicular fossa radiation field treatment

(ii) Central lung distance

Upto $1.5 \mathrm{~cm}$

$1.5-2.5 \mathrm{~cm}$

$2.5-3.5 \mathrm{~cm}$

$>3.5 \mathrm{~cm}$

\section{4) Recurrence pattern}

Recurrence

local recurrences [chest wall]

systemic recurrences

5) Systemic metastasis

Brain
Lung
Liver
Bone
Contralateral breast

\section{6) Acute radiation reaction}

(i) Acute dermatitis grade 1

grade 2

grade 3

grade 4

(ii) Acute esophagitis

grade

grade 2

grade 3

grade 4

(iii)Acute pneumonitis

grade 1

grade 2

grade 3

grade 4
49.6

30.4

-

20

97.7

66

100

135

77

50

75.5

38

2

$-$

15

23

22

1

1.5

1

95

1.5

1.5

1.5

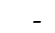

6

14

3

11

8

1.7

6.3

6

4

4

2.3

2.3

1.1

0.6

2

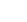

\section{3}

1.5

-

1.5 
Table 4. Relationship between central lung distance and acute pneumonitis using ANOVA test.

\begin{tabular}{ccc}
\hline Grade of acute pneumonitis & Number of patients & Mean CLD (cm) \\
\hline 0 & 220 & 1.15 \\
1 & 10 & 1.25 \\
2 & 7 & 1.64 \\
3 & 4 & 1.55 \\
\hline
\end{tabular}

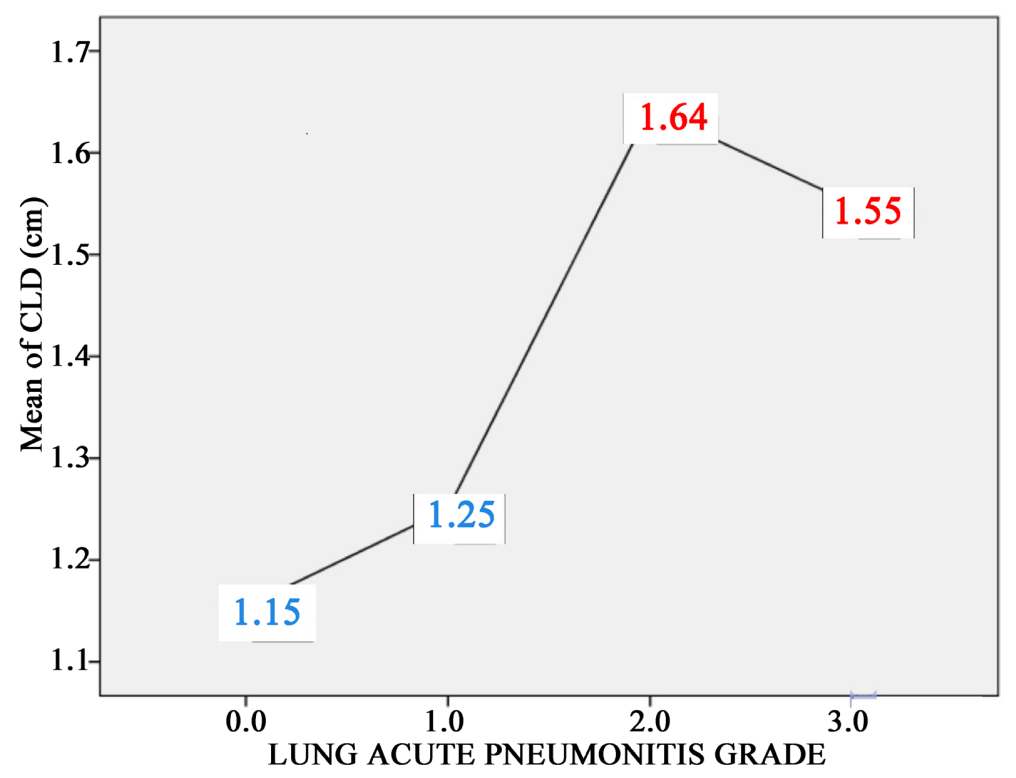

Figure 1. ANOVA test showing relationship between central lung distance and acute pneumonitis.

by Anand AS et al. in which median age in hypofractionation arm was 50 years and in conventional fractionation arm was 46 years [9]. The mean duration of symptoms among patients in hypofractionation arm was 6.23 months (SD-6.38) and in conventional fractionation arm was 8.48 months (SD-10.96). Majority of the patients in both arms had an ECOG performance status of 1 ( $\mathrm{p}$ value $=0.42$ ). Majority of the patients in both arms did not have any comorbid conditions. This observation is similar to that of the study conducted by Anand AS et al. [9]. Majority of the patients in both arms presented with a lump in the breast. The mean age at menarche in patients in the hypofractionation arm was (13.73 \pm 1.16) years and in the conventional fractionation arm was (13.65 \pm 0.98$)$ years. $70.9 \%$ and $50 \%$ of the enrolled patients were postmenopausal in hypofractionation and conventional fractionation arms respectively. This finding has statistical significance ( $\mathrm{p}$ value $=0.01$ ) and is consistent with the findings of the study conducted by Anand AS et al. (69.4\% and 52.2\% patients were postmenopausal in hypofractionation and conventional fractionation, respectively) [9].

In both the arms, majority had a parity of $2: 75.4 \%$ in hypofractionation and $78.8 \%$ in conventional fractionation arm. Adequate breast feeding was practised 
by $98.3 \%$ of patients in hypofractionation arm and $93.9 \%$ in conventional fractionation arm. Majority of the patients in both the arms had no history of oral contraceptive use. Of the 241 patients studied, majority did not affirm a positive family history. Of the $17.1 \%$ patients in hypofractionation arm, who had a family history of malignancy, $4 \%$ had breast cancer in a first degree relative, $2.8 \%$ had the same in a second degree relative. Ovarian cancer, uterine cancer, brain cancer and other malignancies were also reported among family members of the patients in hypofractionation arm. In conventional fractionation arm, $18.1 \%$ of the patients had a positive family history of malignancy, out of which the most common was breast cancer in a first degree relative (7.5\%). In hypofractionation arm, 74 patients (42.3\%) had right sided, and 101 patients (57.5\%) had left sided breast cancer, while in conventional fractionation arm, 32 patients $(48.5 \%)$ had right sided and 34 patients (51.5\%) had left sided breast cancer. In contrast, 66 (76.74\%) patients had right sided and $20(23.25 \%)$ patients had left sided breast cancer in hypofractionation arm, while $9(39.2 \%)$ patients had right sided and 14 (60.8\%) patients had left sided breast cancer, in the study conducted by Anand AS et al. [9].

Most of the patients ( $80 \%$ in hypo fractionated and $82 \%$ in conventional fractionation arms) presented with lump in the upper outer quadrant of the breast. Literature review underscores the fact that upper outer quadrant is the most common quadrant in which breast cancers arise. Majority of the patients had a mammogram finding of BIRADS 5 in both the arms. Most common tumour size status was T2 for both hypofractionation (75 patients - 42.9\%) and conventional arm (28 patients - 42.4\%). In the study by Anand AS et al., most common tumour size status was T3 for hypofractionation arm while it was T2 status in conventional fractionation arm [9]. Most common nodal status was N1 for both hypofractionation (69 patients - 39.4\%) and conventional arm (24 patients $36.4 \%)$. In hypofractionation arm, $40 \%$ was stage II and $54.3 \%$ stage III, while in conventional arm, $31.8 \%$ was stage II and $59.1 \%$ stage III ( $\mathrm{p}$ value $=0.32$ ). In the study conducted by Wang SL et al. published in Lancet Oncology in 2019, 94\% among the 401 patients in the hypo-fractionated radiotherapy group and $94 \%$ of the 409 patients in the conventional fractionated radiotherapy group had stage III disease [13].

Tallying with the international data, in both arms, majority had histology suggestive of invasive ductal carcinoma (95\% in hypofractionation $95.5 \%$ in conventional). This correlates with the observations of the study conducted by Wang SL et al. in which 95\% of the patients in the hypofractionated radiotherapy arm and $93 \%$ of the patients in the conventional fractionated radiotherapy arm had invasive ductal carcinoma [13]. In the study by Anand AS et al. also, the most common histopathology among the patients in both the arms was invasive ductal carcinoma [9]. Majority in both arms had tumour grade 2 (83\% in hypofractionation and 79\% in conventional arm). In the study by Anand AS et al., $100 \%$ of the tumours in both the arms were high grade (either Gr 2 or Gr 3) in both the arms. In our study, almost $100 \%$ of the patients were high grade (grade 
$2 / 3$ ). It is already reported in several Indian studies and also in another study by Anand AS et al., that the disease has high burden and less hormone positivity due to the advanced nature of the disease at presentation [9]. Other tumour charecteristics were similar in the two arms.

45.7\% were ER positive, 29.1\% were PR positive and 24.6\% were HER2-neu positive in hypofractionation arm while in conventional fractionation arm, $34.8 \%$ were ER positive, $18.2 \%$ were PR and $28.8 \%$ were HER2-neu positive. In the study by Anand AS et al., more than $80 \%$ in hypofractionation arm and more than $56 \%$ in conventional fractionation arm were hormone positive (either ER or PR positive) tumour and the incidence of HER2neu positivity in the study arms range between $21 \%$ - 24\% [9]. HER-2/neu gene amplification and protein over-expression has been reported in $20 \%$ - $25 \%$ of cases and was traditionally associated with poor prognosis due to an aggressive tumour phenotype, increased metastasis and poor survival [14]. The Her2neu positivity is $24.5 \%-29 \%$ in our study, which is a little on the higher side when compared to other studies.

Of the total 241 patients enrolled into the study, 175 patients $(72.6 \%)$ received hypofractionated radiation (42.5 Gy in 16 fractions), while 66 patients $(27.3 \%$ ) received conventional fractionation radiation (50 Gy in 25 fractions). In the study by Anand AS et al., of the total 108 patients enrolled for the study, 86 patients $(78.9 \%)$ received hypofractionated radiation while 23 patients $(21.1 \%)$ received conventional fractionation [9]. The major share belonged to the hypofractionated arm, may be due to the physicians being more inclined to give hypofractionated regimen to reduce the fraction numbers and thereby curtailing the long waiting for radiation in the institution and also to reduce the burden for the patients traveling from distant places. The median duration between surgery and the start of radiotherapy in all patients is 6 months, while it is 5.3 months in the study by Wang SL et al. [13].

In hypofractionation arm, 26.3\% received neoadjuvant chemotherapy and $73.7 \%$ received adjuvant chemotherapy, while in conventional fractionation arm, $24.2 \%$ received neoadjuvant chemotherapy and $75.8 \%$ received adjuvant chemotherapy. Most commonly used chemotherapy schedule in both the arms were AC $\times 4$ cycles followed by Docetaxel $\times 4$ cycles with $49.6 \%$ in hypofractionation and $95 \%$ in conventional. In hypofractionation arm, 30.4\% received AC $\times 4 \mathrm{cy}$ cles followed by Paclitaxel $\times 4$ cycles and $20 \%$ received TAC $\times 6$ cycles. In the study by Anand AS et al., the most commonly used chemotherapy schedule in both the arms were FEC for 6 cycles with 74.4\% in hypofractionation arm and $82.5 \%$ in hypofractionation arm; $22.1 \%$ in hypofractionation arm and $4.3 \%$ in conventional fraction arm received $\mathrm{AC} \times 4$ followed by Paclitaxel $\times 4$; remaining set of patients received TAC $\times 6$ cycles [9]. Almost all the patients in both arms were treated with a supraclavicular fossa field. Other radiation planning details like mean depth of radiation treatment, mean central lung distance, mean $\mathrm{X}$ for treatment, mean Y for treatment and mean IFD were similar between the two groups. Mean follow up period of all the patients taken together was 8 months, while it was 56 months in the study by Anand AS et al. [9] and 58.5 months in 
the study by Wang SL et al. [13].

\section{a) Recurrence Rate}

In hypofractionation arm, recurrence was found in 14 patients (8\%), out of which 3 were local recurrences [chest wall] and the rest were systemic metastasis. Among the systemic metastasis, most common sites were brain and lung (4 patients each) and next most common site was liver ( 2 patients) followed by bone (1 patient); no contralateral breast metastasis were found. In conventional arm, recurrence was found in 4 patients (6\%). All 4 were systemic recurrences; most common site was lung ( 2 patients) and next most common site was liver and contralateral breast (1 patient each); no brain or bone metastasis was found. In the study by Anand AS et al., 27 patients in the hypofractionated arm (31.4\%) and $6(26.1 \%)$ patients in the conventional arm developed recurrence. Of the recurrences, $10(11.6 \%)$ in hypofractionation arm and $2(8.7 \%)$ in conventional arm were loco-regional recurrence, while 17 (19.8\%) and 4 (17.4\%) had systemic relapse respectively [9]. In hypofractionation arm, most common site of loco regional recurrence was drainage areas, while it is chest wall in conventional fractionation. The most common site of systemic metastases was liver (9 patients) in hypofractionation arm and lung (2 patients) in conventional arm. Lung, brain and bone were the next common sites in hypofractionation arm, while bone and brain in conventional arm [9].

Recurrence rate in hypofractionation arm was $8 \%$ and in conventional fractionation arm was $6.10 \%$. The Kaplan Meier curve of recurrence rate shows no significant difference between the two arms ( $\mathrm{p}$ value $=0.76$ ).

\section{b) Acute Radiation Toxicities}

The acute toxicities assessed were dermatitis, esophagitis and pneumonitis. Cutaneous toxicity was mostly grade 1 (10.3\% in hypofractionation arm and $28.8 \%$ in conventional arm). Grade 2 dermatitis was $5 \%$ in hypofractionation arm and $10.6 \%$ in conventional arm. 4 patients (2.2\%) had grade 3 in hypofractionation arm while 7 patients (10.6\%) in conventional arm. 2 patients had grade 4 in hypofractionation and 1 patient had grade 4 in conventional. This difference in incidence is statistically significant with $\mathrm{p}$ value $\leq 0.01$. In the study by Anand AS et al., the assessed acute toxicities was cutaneous toxicity and it was mostly grade 1 (83\% in hypofractionation arm and $81 \%$ in conventional arm). The incidence of grade 2 acute dermatitis was $9.8 \%$ in hypofractionation arm and $11.7 \%$ in conventional arm and no patients experienced grade 3 acute dermatitis [9]. In the study by Wang SL et al., grade 1 - 2 acute dermatitis was found in 351 patients $(89 \%)$ in hypofractionation arm and 357 patients $(87 \%)$ in conventional arm; while grade 3 acute dermatitis was found in 14 patients (3\%) in hypofractionation arm and 32 patients (8\%) in conventional arm [13].

In hypofractionation arm, 15 patients (8.6\%) had grade 1 acute esophagitis, 15 patients (8.6\%) had grade 2 acute esophagitis and 1 patient had grade 3 . In conventional arm, 6 patients $(9.1 \%)$ had grade 1,1 patinet had grade 2 . No grade 4 were reported. In hypofractionation arm, 6 patient (3\%) had grade 1 acute pneumonitis, 6 patients (3\%) had grade 2, 2 patients had grade 3 and 1 patient 
had grade 4 . In conventional arm, 4 patient (6\%) had grade 1, 1 patinet had grade 2 and 1 patient had grade 3 . No grade 4 were reported. In the study by Wang SL et al., in hypofractionation arm, 61 patients (15\%) developed grade 1 pneumonitis and 14 patients (3\%) developed grade 2 pneumonitis; while in conventional arm, 62 patients (15\%) developed grade 1 pneumonitis and 7 patients (2\%) developed grade 2 pneumonitis [13]. Of the 241 patients, those with grade 1 acute pneumonitis had a mean CLD of $1.25 \mathrm{~cm}$. Grade 2 and grade 3 acute pneumonitis were found in patients with central lung distance more than $1.5 \mathrm{~cm}$. (grade 2-mean CLD $1.64 \mathrm{~cm}$; grade 3-mean CLD $1.55 \mathrm{~cm}$ ).

\section{c) Limitations of the study}

The sample size calculated prior to the study was 410 patients each in hypofractionation and conventional fractionation arms, based on the reference study conducted by Anand AS et al. [9]. We were able to recruit only 241 patients who satisfied the selection criteria. 175 patients were included in the hypofractionation arm and 66 patients in the conventional fractionation arm. This may be due to the reason that (a) the patients who completed the planned treatment were less in number and (b) patients were lost follow up. Our study was conducted as prospective observational and hence had its own limitations compared to a randomised control trial. The duration of follow up for our study was a period of 18 months, which is too short. Hence, it is difficult to apply the results of this study into clinical practice.

\section{Conclusions}

Local radiation treatment is an integral treatment modality in breast cancer. Conventional fractionation was widely used for past several decades, but due to the low alpha/beta value, hypofractionation is studied and now it is the radiation of choice after BCS. But data for hypofractionation is less in the postmastectomy setting. Our study compared conventional fractionation versus hypofractionation in breast cancer patients in the postmastectomy setting.

This study included 241 patients, of which 175 patients received 42.5 Gy in 16 fractions (hypofractionation arm) and 66 patients received 50 Gy in 25 fractions (conventional fractionation arm). Recurrence rate in hypofractionation arm was $8 \%$ and in conventional fractionation arm was $6.10 \%$. There was no significant difference between the recurrence rates between the two arms. Toxicities assessed in the two arms were acute dermatitis, acute esophagitis and acute pneumonitis. There was no significant difference between acute esophagitis and acute pneumonitis between the two arms. Acute dermatitis showed a significant difference, with grade 1 and grade 2 acute dermatitis more in the conventional fractionation arm. It is thus observed that the loco-regional control of the disease is similar in both hypofractionated and conventional fractionation radiation arms with comparable toxicity.

Our study is conducted as prospective observational and has its own limitations compared to a randomised control trial. Randomised control trials study- 
ing hypofractionation radiation in postmastectomy breast cancer patients are very less in number. Hence, our study results will be an eye opener for further randomised trials, especially in developing countries like India, where the resources are limited and the incidence of breast cancer is high.

\section{Conflicts of Interest}

The authors declare no conflicts of interest regarding the publication of this paper.

\section{References}

[1] Lawrence, V.T., Rosenberg, T.S. and Devita, S.A. (2019) Hellman \& Rosenberg's Cancer: Principles \& Practice of Oncology, 10th Edition.

[2] Bentzen, S.M., Agrawal, R.K., et al. (2008) START Trialists' Group. The UK Standardisation of Breast Radiotherapy (START) Trial A of Radiotherapy Hypofractionation for Treatment of Early Breast Cancer: A Randomised Trial. Lancet Oncology, 9, 331-341. https://doi.org/10.1016/S1470-2045(08)70077-9

[3] Bentzen, S.M., Agrawal, R.K., et al. (2008) START Trialists' Group. The UK Standardisation of Breast Radiotherapy (START) Trial B of Radiotherapy Hypofractionation for Treatment of Early Breast Cancer: A Randomised Trial. Lancet, 371, 1098-1107. https://doi.org/10.1016/S0140-6736(08)60348-7

[4] Haviland, J.S., Owen, J.R., et al. (2013) The UK Standardisation of Breast Radiotherapy (START) Trials of Radiotherapy Hypofractionation for Treatment of Early Breast Cancer: 10-Year Follow-Up Results of Two Randomised Controlled Trials. Lancet Oncology, 14, 1086-1094. https://doi.org/10.1016/S1470-2045(13)70386-3

[5] Ko, D.H., et al. (2015) Hypofractionated Radiation Treatment Following Mastectomy in Early Breast Cancer: The Christchurch Experience. Journal of Medical Imaging and Radiation Oncology, 59, 243-247. https://doi.org/10.1111/1754-9485.12242

[6] Ortholan, C., Hannoun-Lévi, J.-M., et al. (2005) Long-Term Results of Adjuvant Hypofractionated Radiotherapy for Breast Cancer in Elderly Patients. International Journal of Radiation Oncology, Biology, Physics, 61, 154-162. https://doi.org/10.1016/j.ijrobp.2004.04.059

[7] Khan, A.J., et al. (2017) Hypofractionated Postmastectomy Radiation Therapy Is Safe and Effective: First Results from a Prospective Phase II Trial. Journal of Clinical Oncology, 35, 2037-2043. https://doi.org/10.1200/JCO.2016.70.7158

[8] Whelan, T., MacKenzie, R., et al. (2002) Randomized Trial of Regional Irradiation Schedules after Lumpectomy for Women with Lymph Node-Negative Breast Cancer. Journal of the National Cancer Institute, 94, 1143-1150. https://doi.org/10.1093/jnci/94.15.1143

[9] Anand, A.S., et al. (2017) Hypofractionated vesus Conventional Fractionation in Postmastectomy Adjuvant Radiation in Breast Cancer. Department of Radiation Oncology, GMC, Tvm.

[10] Whelan, T.J., Pignol, J.P., Levine, M.N., Julian, J.A., et al. (2010) Long Term Results of Hypofractionated Radiation Therapy for Breast Cancer. New England Journal of Medicine, 362, 513-520. https://doi.org/10.1056/NEJMoa0906260

[11] Yarnold, J., Ashton, A., et al. (2005) Fractionation Sensitivity and Dose Response of Late Adverse Effects in the Breast after Radiotherapy for Early Breast Cancer: 
Long-Term Results of a Randomised Trial. Radiotherapy \& Oncology, 75, 9-17. https://doi.org/10.1016/j.radonc.2005.01.005

[12] Owen, J.R., Ashton, A., et al. (2006) Effect of Radiotherapy Fraction Size on Tumour Control in Patients with Early-Stage Breast Cancer after Local Tumour Excision: Long-Term Results of a Randomised Trial. Lancet Oncology, 7, 467-471. https://doi.org/10.1016/S1470-2045(06)70699-4

[13] Wang, S.L., et al. (2019) Hypofractionated versus Conventional Fractionated Postmastectomy Radiotherapy for Patients with High-Risk Breast Cancer: A Randomised, Non-Inferiority, Open-Label, Phase 3 Trial. Lancet Oncology, 20, 352-360. https://doi.org/10.1016/S1470-2045(18)30813-1

[14] Emde, A., Köstler, W.J., et al. (2012) Therapeutic Strategies and Mechanisms of Tumourigenesis of HER2-Overexpressing Breast Cancer. Critical Reviews in Oncology/Hematology, 84, e5. https://doi.org/10.1016/j.critrevonc.2010.09.002 